\title{
GEOGRAFIA E MÚSICA: A DUPLA FACE DE UMA RELAÇÃO
}

- DANIEL DE CASTRO'

\section{RESUMO}

ESTE ARTIGO DISCUTE ALGUNS DOS PRINCIPAIS TRABALHOS QUE ABORDARAM UMA POSSÍVEL INTERFACE ENTRE A GEOGRAFIA CULTURAL E A MÚSICA NOS ÚLTIMOS QUARENTA ANOS. ADICIONALMENTE, APRESENTA AS CONTRIBUIÇÕES DOS DOIS PRINCIPAIS AUTORES QUE SE DEBRUÇARAM SOBRE ESTE TEMA, ARTICULANDO-AS AOS DEBATES ENTRE A GEOGRAFIA CULTURAL TRADICIONAL E A GEOGRAFIA CULTURAL RENOVADA.

PALAVRAS-CHAVE: GEOGRAFIA CULTURAL. MÚSICA POPULAR. GEORGE CARNEY, LILY KONG.

CONSIDERAÇÕES INICIAIS

As transformações que a geografia cultural sofreu a partir dos anos 70 trouxeram novas matrizes epistemológicas e metodológicas para a disciplina, gerando um debate considerado por alguns como uma "dicotomia" entre a geografia cultural tradicional e a new cultural geograpby, ou geografia cultural renovada. Esse debate, além de permitir a inclusão de novos objetos de estudo ao "repertório" da geografia cultural, permite novas acepções e abordagens a objetos que já eram de interesse da disciplina anteriormente. Os estudos geográficos sobre música, ou music geography, exemplificam muito bem esta situação. O objetivo do presente artigo é apresentar uma breve retrospectiva deste sub-campo de estudo, apontando os principais trabalhos e eventos realizados no exterior, assim como as iniciativas de geógrafos brasileiros interessados no tema. Além disso, serão apresentadas as contribuições dos dois autores mais importantes no que diz respeito a esta área de estudo: George O. Carney e Lily Kong. Importantes no sentido de possuírem o maior número de publicações a respeito do tema e por terem se dedicado a um esforço de sistematizar suas diversas vertentes. Por fim, será empreendida uma breve análise comparativa das diferentes contribuições destes autores e de como elas estão inseridas na discussão sobre a dita "dicotomia" entre a geografia cultural tradicional e a geografia cultural renovada.

\section{UMA TRAJETÓRIA DE QUATRO DÉCADAS}

Um dos mais importantes trabalhos já realizados na área de geografia e música é de autoria de 
George O. Carney, no livro The Sounds of People and Places: Readings in the Geograpby of Music, originalmente lançado em 1978, no qual o autor apresenta uma coletânea de ensaios próprios e de outros autores que se dedicaram a este tema. $\mathrm{Na}$ introdução da sua quarta edição, de 2003, Carney expõe uma detalhada retrospectiva da história deste ramo da geografia cultural, desde o primeiro artigo sobre música escrito por um geógrafo, até as tendências mais recentes. É a partir desse trabalho que se baseia grande parte do presente estudo.

A trajetória dos estudos de geografia sobre música inicia-se há quarenta anos, com o artigo de Peter Hugh Nash, "Music Regions and Regional Music", de 1968. Apesar de ser uma tradição relativamente longa, ela permaneceu praticamente restrita à geografia norte-americana durante quase todo esse tempo, com raras exceções que veremos mais adiante. Em 1970, é concluída a primeira Master's thesis sobre o tema: Rock-and-Roll: A Diffusion Study, de Jeffrey Gordon. Em 1971, o primeiro artigo integral, de autoria de Larry Ford, Geographic Factors in the Origin, Evolution and Diffusion of Rock and Roll Music, aparece no periódico americano Journal of Geograpby. A partir de então, uma série de trabalhos são publicados até que, em 1974, na primeira SNACS (Society for the North American Cultural Survey) , a Música foi designada como um dos capítulos para This Remarkable Continent: An Atlas of United States and Canadian Society and Cultures, que foi publicado em 1982. Após essa publicação, os estudos musicais passaram a ser considerados como um novo sub-campo dentro da geografia cultural (Carney, 2003).

A partir dos anos 1980, sessões especiais sobre música têm sido realizadas nas reuniões anuais da
Association of American Geographers em diversas cidades, San Antonio (1982), Detroit (1985), Minneapolis (1986), San Diego (1992), Atlanta (1993), San Francisco (1994), Chicago (1995), Charlotte (1996), Fort Worth (1997), Boston (1998), Honolulu (1999), Pittsburgh (2000), New York (2001) e Los Angeles (2002). A expansão desse sub-campo ultrapassa as fronteiras dos Estados Unidos. Em 1993, uma reunião intitulada "Place of Music" foi realizada no University College London, sob o patrocínio dos Economic Geograpby, Landscape, and Social/Cultural Geograpby Research Groups (Carney, 2003). A partir desse evento, origina-se mais um dos grandes trabalhos realizados sobre o tema, o texto Popular Music in Geograpbical Analyses, da autora cingapuriana Lily Kong, que será discutido mais adiante.

Diversos artigos foram publicados em diferentes revistas acadêmicas americanas, como o Journal of Cultural Geography, The Professional Geographer, The Geographycal Review e o Journal of Geography, além dos não-americanos, como Transactions of the Institute of British Geographers, The Canadian Geographer e o Progress in Human Geograpby (Carney, 2003). A revista francesa Géograpbie et Cultures, fundada por Paul Claval, publicou em 2006 um número dedicado ao tema, entitulado GÉOGRAPHIES ET MUSIQUES: Quelles perspectives?, com uma coletânea de textos de diversos geógrafos, com abordagens que vão desde o estudo do perímetro espacial e musical da ópera em Provença, até o estudo da articulação entre a lógica global e as características locais a partir do bip-bop na Mongólia.

Em 2003, é publicado um dos mais completos e atualizados trabalhos sobre o tema. Trata-se do livro dos geógrafos, John Connell e Chris Gib- 
son, da University of Sydney, intitulado Sound tracks. Popular music, identity and place. A fotografia da capa, uma banda de flauta de pã, típica de países andinos, apresentando-se na calçada da Times Square, em Nova York, retrata o teor do texto, que aborda a espacialidade da música popular, mais especificamente, focalizando a relação entre música e mobilidade espacial, as formas pelas quais a música está ligada aos elementos culturais, étnicos e geográficos da identidade e como estas questões estão atreladas às transformações de ordem econômica, tecnológica e cultural (Connell \& Gibson, 2003).

No Brasil, poucos trabalhos foram realizados sobre geografia e música, mas podemos destacar a pioneira dissertação de mestrado de Mello (1991) sobre composições da MPB e o Rio de Janeiro; o artigo de Mesquita (1997) sobre a Geografia Social na música do Prata; a tese de doutorado de Ribeiro (2006), abordando o conceito de espaço-vivo e suas variáveis na cidade de Diamantina sob o ponto de vista dos músicos; e a dissertação de mestrado de Marcelino (2007), que aborda as transformações sofridas pelo samba paulista em sua transição da zona rural, concentrado principalmente na cidade de Pirapora do Bom Jesus, para a metrópole de São Paulo a partir do final do século XIX e durante o século XX. Em 2000, foi publicado um excelente artigo do antropólogo Pessoa de Barros (2000), no qual ele busca a compreensão dos rituais do candomblé, a história dos mitos e dos ritos, a partir dos seus cantos litúrgicos. Segundo ele (2000, p. 35-36), "Dedicamos especial atenção a estes cânticos como também aos diferentes ritmos que os acompanham, pois julgamos serem eles parte significativa na manutenção da memória africana no Brasil". Portanto, o autor deixa claro que a sua análise envolve alguns dos elementos básicos que compõem a música (melodia e ritmo), e não somente uma análise dos textos cantados. Ele aborda também a importância de cada instrumento musical que compõe a orquestra, assim como as funções que são acumuladas pelo alabê, figura equivalente ao maestro das orquestras convencionais. Entretanto, a análise também não se limita a somente descrever as características fundamentais da música sacra do candomblé e, sim, relaciona essas características com as outras manifestações não-musicais, assim como a sua função de transmitir os valores da comunidade, reforçando a sua identidade:

Nesse mundo de sons, os textos, falados ou cantados, assim como os gestos, a expressão corporal e os objetos-símbolo transmitem um conjunto de significados determinado pela sua inserção nos diferentes ritos. Reproduzem a memória $e$ a dinâmica do grupo, reforçando e integrando os valores básicos da comunidade, através da dramatização dos mitos, da dança e dos cantos, como também nas bistórias contadas pelos mais velhos como modelos paradigmáticos (PESSOA DE BARROS, 2000, p.37)

Há que se destacar também a valiosa contribuição do Núcleo de Estudos e Pesquisas sobre Espaço e Cultura (NEPEC), da UERJ. Na revista Espaço e Cultura, foram publicados textos sobre o tema como o de Corrêa (1998), além dos já mencionados Mesquita (1997) e Pessoa de Barros (2000). Em 2006, o NEPEC realizou o $5^{\circ}$ Simpósio Nacional e $1^{\circ}$ Internacional sobre Espaço e Cultura no qual 
uma das mesas redondas foi denominada "Espaço, Literatura e Música", com a apresentação de trabalhos de geógrafos brasileiros interessados em desbravar esse sub-campo. E em 2007, a $14^{\text {a }}$ publicação da Coleção Geografia Cultural, é intitulada Literatura, Música e Espaço, com textos de Marc Brosseau sobre o viés literário, e de George O. Carney, a respeito do viés musical.

Atualmente, pode-se considerar George O. Carney e Lily Kong como os dois autores mais importantes na área de Geografia e Música. Ambos publicaram não apenas trabalhos empíricos que abordam a atividade musical dos Estados Unidos e Cingapura, respectivamente, sobre a ótica espacial, mas também desenvolveram análises sobre este sub-campo de estudo, cada qual apresentando diferentes linhas de pesquisa já exploradas pelos geógrafos e, além disso, oferecendo propostas de agendas de pesquisa para os novos geógrafos que se interessam pelo tema.

\section{A CONTRIbuição DE GeOrGe O. CARNEY}

Em um artigo de 1996, Nash e Carney oferecem uma sistematização na qual sete temas principais são delineados na formação da music geography, para servirem de referência aos geógrafos interessados pelo tema: I - origens (não-geográficas); II - distribuição mundial e tipos, III - análises de localização; IV - áreas de origem de atividades musicais; $\mathrm{V}$ - tendências baseadas em eletricidade; VI - impacto nas paisagens; VII - música global. Os autores fariam ainda referência a um oitavo tema emergente, inovações tecnológicas, ainda carente de estudos (Nash \& Carney, 1996). Em função do crescimento e desenvolvimento deste sub-campo nos anos 90, esta sistematização sofreria gran- des transformações posteriormente, tornando-se mais complexa, como se pode comprovar no primeiro capítulo da quarta edição de The Sounds of People and Places, no qual Carney articula nove fenômenos musicais observáveis com dez temas passíveis de abordagem pela geografia. Segundo Carney (2003), os fenômenos musicais sobre os quais os geógrafos têm se debruçado dividem-se em nove categorias gerais: (1) estilos/gêneros, (2) estrutura, (3) letras, (4) instrumentação, (5) intérpretes e compositores, (6) centros e eventos, (7) mídia, (8) música étnica e (9) indústria. Na geografia norte-americana, mais da metade dos trabalhos são dedicados à música country, seus vários sub-estilos, letras e instrumentação, 20\% são voltados para o Rock and Roll, e o restante explora uma grande variedade de gêneros, como a música clássica, gospel, jazz, blues, música folclórica, étnica, entre outros.

A partir destas nove categorias de fenômenos musicais observáveis, os geógrafos exploram uma diversidade de abordagens e temas, que podem ser agrupados em dez tipos gerais (Carney, 2003):

1. A delimitação de regiões musicais e a interpretação da música regional, como os sub-estilos do country no Sul dos Estados Unidos, o Reggae na Jamaica e a música Cajun do sul da Louisiana.

2. A evolução de um estilo musical com o lugar, ou a música de um lugar-específico, por exemplo: Viena e o clássico, Nashville e o country, Detroit e Motown, Seattle e o Grunge, a 52nd Street de Nova York e o bebop jazz.

3. A origem e a difusão do fenômeno musical, como na trajetória da difusão do country blues, da sua origem, no delta do Mississipi, para Chicago 
com os músicos do blues atuando como agentes de difusão.

4. A relação entre a distribuição espacial da música e as migrações humanas, rotas de transporte e redes de comunicação como, por exemplo, na transnacionalização da música a partir do intercâmbio de artistas entre os países, além da importação e exportação de LPs, K7s e CDs, assim como as trocas de músicas em formato MP3 pela Internet, que resultam na popularidade de gêneros norte-americanos no restante do mundo.

5. Os elementos psicológicos e simbólicos da música moldando o caráter de um lugar: a imagem do lugar, o sentido de lugar e a percepção do lugar. Pode-se citar o trabalho de Lechaume (1997), que examina a influência que a chanson exerceu na evolução da identidade territorial do Quebec.

6. Os efeitos da música na paisagem cultural: salas de concertos, festivais de rock e mega-shows.

7. A organização espacial da indústria fonográfica e outros fenômenos musicais.

8. A relação da música com o ambiente natural. Por exemplo, um concerto ao ar livre em área não-urbana, o uso da madeira na construção de flautas indígenas, a representação de aspectos naturais da paisagem na música: ventos, canto de pássaros, tempestades.
9. A função da música "nacionalista" e "antinacionalista". De um lado, compositores que, em sua obra, deram destaque aos valores nacionais, às vitórias, às belezas e ao folclore, como no caso de Antonín Dvorák, Bela Bartók e Heitor Villa-Lobos. De outro, sentimentos antipatrióticos retratados por bandas de punk rock como os britânicos Sex Pistols, em músicas como "God Save the Queen" e "Anarchy in the UK".

10. As interrelações da música com outros traços culturais em um sentido espacial, por exemplo, a religião, as gírias, a política, a culinária e os esportes.

Carney (2003), utilizando uma matriz desenvolvida pelo autor Ed Huefe (quadro 1), combina estas dez abordagens de pesquisa com as nove categorias musicais apresentadas anteriormente. $\mathrm{O}$ objetivo é oferecer uma síntese da ampla gama de possibilidades existentes no campo da geografia e música, que podem ser caracterizadas sob diversos adjetivos: empírica, descritiva, humanista, ateorética, não-analítica e subjetiva, pois elas flertam com teorias e métodos que vão desde a análise quantitativa e o difusionismo, que se aproximam da escola saueriana, até conceitos utilizados pela "nova" geografia cultural, como o espaço vivido e a paisagem simbólica, por exemplo. 
Quadro 1: A proposição de G. O. Carney para o estudo da música Pela Geografia

\begin{tabular}{|c|c|c|c|c|c|c|c|c|}
\hline \multirow[b]{2}{*}{ Abordagens de Pesquisa } & \multicolumn{8}{|c|}{ Categorias Musicais } \\
\hline & Estilos & Estrutura & Letras & $\begin{array}{c}\text { Compositores } \\
\text { / Eventos }\end{array}$ & Mídia & Etnias & Instrumentação & Indústria \\
\hline \multirow{2}{*}{\multicolumn{9}{|c|}{$\begin{array}{l}\text { Regiões Musicais e Música } \\
\text { Regional }\end{array}$}} \\
\hline & & & & & & & & \\
\hline \multirow{2}{*}{\multicolumn{9}{|c|}{$\begin{array}{l}\text { Evolução da Música e Seu Lugar } \\
\text { Específico }\end{array}$}} \\
\hline & & & & & & & & \\
\hline \multirow{2}{*}{\multicolumn{9}{|c|}{$\begin{array}{l}\text { Origem e Difusão de um } \\
\text { Gênero Musical }\end{array}$}} \\
\hline & & & & & & & & \\
\hline \multirow{3}{*}{\multicolumn{9}{|c|}{$\begin{array}{l}\text { Dimensão Espacial da Música } \\
\text { em Relação A Migrações, } \\
\text { Transportes e Comunicação }\end{array}$}} \\
\hline & & & & & & & & \\
\hline & & & & & & & & \\
\hline \multirow{2}{*}{\multicolumn{9}{|c|}{$\begin{array}{l}\text { Elementos Simbólicos e o } \\
\text { Caráter do Lugar }\end{array}$}} \\
\hline & & & & & & & & \\
\hline \multirow{2}{*}{\multicolumn{9}{|c|}{$\begin{array}{l}\text { Efeitos da Música na Paisagem } \\
\text { Cultural }\end{array}$}} \\
\hline & & & & & & & & \\
\hline \multirow{2}{*}{\multicolumn{9}{|c|}{$\begin{array}{l}\text { Organização Espacial da } \\
\text { Indústria Fonográfica }\end{array}$}} \\
\hline & & & & & & & & \\
\hline \multicolumn{9}{|l|}{ Relação da Música com o } \\
\hline \multicolumn{9}{|l|}{ Ambiente Natural } \\
\hline \multicolumn{9}{|l|}{ Música Nacionalista e } \\
\hline \multicolumn{9}{|l|}{ Antinacionalista } \\
\hline \multicolumn{9}{|l|}{ Inter-Relação entre Aspectos } \\
\hline Espaciais da Música e Outros & & & & & & & & \\
\hline Traços Culturais & & & & & & & & \\
\hline
\end{tabular}

(EXTRAíDA DE CARNEY, 2003, P.6)

\section{A CONTRIBUIÇÃO DE LILY KONG}

Entre os mais importantes trabalhos já realizados a respeito de geografia e música, está o de Kong (1995), intitulado Popular music in geograpbical analyses. A autora se propõe a focalizar a interface existente entre geografia e música, visando à contribuição que esse tipo de pesquisa pode dar ao entendimento cultural e social. Segundo Lily Kong, a relativa negligência dos geógrafos em relação à pesquisa com música, dentre outras ma- nifestações artísticas, está baseada em dois pontos principais. Em primeiro lugar, ela afirma que os geógrafos foram, durante muito tempo, "profundamente elitistas" em seus interesses, ou seja, privilegiou-se em demasia a cultura das elites em detrimento da cultura popular, que foi tratada, segundo ela, "com desdém, como mero entretenimento, trivial e efêmero" (KONG, 1995, p. 184). Todavia, essa hegemonia da cultura dominante tem sido contestada recentemente, com o reconheci- 
mento de que a cultura popular é, na verdade, uma fonte inesgotável de consciência popular.

Em segundo lugar, mas não menos importante, está o fato de que a pesquisa geográfica cultural privilegiou em larga escala o estudo de aspectos visuais. Indo ao encontro desta idéia, Smith (1997) argumenta que a geografia humana está envolvida com uma política cultural que, quer explorando o legado do iluminismo (ver é acreditar)ou os presságios do pós-modernismo (imagem é tudo), permaneceu mergulhada na ideologia visual. Os sentidos de olfato, tato, paladar e audição têm sido negligenciados como uma consequência da ênfase na visão. De fato, ainda hoje muitos geógrafos definem paisagem, por exemplo, como "a porção visível do espaço", ou "tudo aquilo que se vê", como se na paisagem também não existissem sons, cheiros ou sabores. O olfato e o paladar, por exemplo, podem evocar um sentido de lugar radicalmente distinto da visão. A autora atenta ainda para a importância dos sons que caracterizam diferentes espaços como o urbano e rural, os naturais, como o canto dos pássaros ou o som do vento nas árvores, e os originados pela atividade humana, como o som de um engarrafamento, ou da confusão de vozes no meio de uma multidão (Kong, 1995).

Para a autora, uma variedade de razões poderia ser citada para justificar o estudo geográfico da música. Uma delas é a de que, hoje, a difusão da música na sociedade é excepcional. Não existe uma sociedade em que não haja música. A música está presente no cotidiano das pessoas, mesmo que servindo apenas como "trilha sonora" para atividades como o trabalho, as compras no supermercado, atividades esportivas, de lazer, cerimônias, ri- tuais religiosos, etc. Ou seja, a música é capaz de transmitir "imagens" de um lugar, podendo servir como fonte primária para entender o caráter e a identidade dos lugares.

Lily Kong ratifica essa idéia e afirma que a música pode servir como um meio, um veículo, através do qual as pessoas transmitem suas experiências ambientais, seja do cotidiano ou de um fato extraordinário, sendo útil para enriquecer discussões que envolvem noções como "espaço", e "lugar". Pode-se acrescentar, como exemplo, o funk de morro carioca. Além disso, a música pode ter o caráter de resultado, produto da experiência ambiental. Um compositor escreve suas músicas inspirado, muitas vezes, em experiências individuais, na memória coletiva de sua comunidade ou seu povo. Um bom exemplo disso é a famosa Abertura 1812, do compositor russo Piotr Il'yich Tchaikovsky, uma obra orquestral que comemora o fracasso da invasão francesa à Rússia e a destruição da Grande Armada de Napoleão Bonaparte. Essa transmissão de sentimentos sobre o lugar pela música pode ser intensa a tal ponto de determinadas obras se tornarem símbolo da identidade de uma nação com o seu território. Exemplo disso é a tão debatida influência de Richard Wagner sobre a ideologia de Adolf Hitler, ou a música de Chopin como símbolo da resistência dos poloneses diante do nazismo, ou mesmo a importância dos hinos nacionais de cada país. Sejam quais forem os objetivos que movem a composição, a maneira através da qual esses sentimentos são expressos varia no tempo e no espaço.

KONG (1995) aponta, ainda, cinco tendências principais para as pesquisas geográficas já realizadas com música. As três primeiras refletem a tra- 
dição da geografia cultural de Berkeley, enquanto as duas últimas estariam mais identificadas com a "nova" geografia cultural. Em primeiro lugar, estão aquelas que buscam demonstrar a distribuição espacial de formas musicais, atividades, artistas e personalidades, sendo essa forma de trabalho originada nos Estados Unidos, como se exemplifica com as obras de autores como Crowley e Carney. Esses trabalhos, segundo ela, não possuem um grande embasamento teórico. Visam apenas ao mapeamento e não abordam os contextos sociopolíticos que acabaram por gerar a predominância de um determinado estilo musical em um determinado lugar.

Um segundo grupo pode ser caracterizado como aquele que visa à exploração dos locais de origem musical e a sua difusão, usando conceitos como contágio, relocação e difusão hierárquica. Procura também verificar os agentes e barreiras para essa difusão. Esta linha de trabalho, segundo a autora, já possui um maior aprofundamento teórico, articulando o objeto de estudo com o contexto em que ele está inserido, além de contribuir com discussões sobre a dinâmica espacial do desenvolvimento musical.

A terceira tendência é a que busca delimitar áreas que partilham certos traços musicais, sendo que essa delimitação ocorre em várias escalas, tais como a global e a regional. O problema que pode surgir nesse tipo de análise, segundo Kong, é a tendência de se tratar a cultura como algo homogêneo, isolando um traço cultural particular e definindo o caráter de uma área apenas com essa base, ignorando as condições sociopolíticas que influenciaram o desenvolvimento daquela característica cultural e ignorando também os con- flitos e tensões existentes com outras culturas na mesma região.

A quarta tendência descrita por Lily Kong é aquela na qual se investiga o caráter e a identidade dos lugares a partir das letras das canções. A autora cita como exemplo o texto de Jarvis, que identificou vários temas nas letras de canções de rock, e Marcus, em sua exploração de imagens da América no rock and roll. Pode-se incluir aqui também os já mencionados trabalhos de Mello (1991) e Mesquita (1997) além de Vilanova Neta (2002).

$\mathrm{Na}$ quinta e última tendência os objetivos são basicamente os mesmos da anterior, ou seja, explorar a relação de identidade dos compositores com o seu espaço. Entretanto, a pesquisa visa a interpretar as características dessa "visão de mundo" expressas através da melodia, instrumentação, letras (eventualmente) e as sensações ou impactos sensoriais transmitidos pela música.

Alem de delinear estas cinco principais vertentes exploradas no campo de geografia e música, Lily Kong propõe novas possibilidades de abordagens, que poderiam ser exploradas por geógrafos e não-geógrafos. As propostas que se seguem, conforme ela própria aponta, estão fundamentadas nas reflexões teóricas que conduziram, nas últimas décadas, ao reposicionamento da geografia cultural, que passa a se preocupar menos com as formas materiais e mais com os significados e valores simbólicos atribuídos a elas, além de levantar questões acerca da produção, circulação e consumo desses, da política cultural e as relações de poder e a teoria da construção social (Kong, 1995):

1. A análise de significados simbólicos. Ou seja, a preocupação com os simbolismos utiliza- 
dos na música, além do papel simbólico da música na vida social. Como exemplo, pode-se mencionar a forma como a música country evoca uma nostalgia do paraíso, de um modo de vida mais simples, sem complicações.

2. Música como comunicação cultural. Baseandose em conceitos como discurso, texto e metáfora, os "textos musicais" devem ser entendidos como diálogos sociais, processos de comunicação que refletem o contexto sociohistórico no qual estão inseridos.

3. A política cultural da música. A questão central aqui é focalizar os produtores de música, as condições sociais, políticas e econômicas nas quais eles atuam e como estas interferem nas suas intenções, seja para perpetuar uma ideologia, contestá-la, ou simplesmente para obter lucro, por exemplo.

4. Economia musical. Focalizando especificamente a indústria fonográfica, a preocupação nesta proposta dirige-se para os aspectos econômicos subjacentes à produção musical: criação de empregos, exportação, propaganda e atração de investimentos.

5. Música e a construção social de identidades. Preocupa-se aqui com o papel da música na construção e desconstrução de identidades nacionais, de gênero, étnicas ou religiosas, evidenciando a importância dessa arte na formação do que Benedict Anderson (1983) chama de "comunidades imaginadas". A título de exemplo, pode-se citar os textos de Hudson (2006), sobre as relações entre música, identidade e lugar, e de Connell e Gibson (2004), nos quais analisam a ascenção e expansão da chamada world music, no final dos anos 80, como um fenômeno comercial ,através do qual os discursos sobre o "global" e o local são produzidos e disseminados.
6. Métodos de análise. Nessa proposta, Lily Kong denuncia uma inadequação nos métodos que vêm sendo utilizados pelos geógrafos: análise de letras, levantamentos de opinião, mapeamentos, etc. Para a autora, abordagens qualitativas e quantitativas podem contribuir de diferentes formas, mas é fundamental que o estudo não se limite a ser um exercício dos acadêmicos visando a impor um ponto de vista dominante para a obra. Para tentar contornar este problema, ela propõe entrevistas com todos os envolvidos na produção de música, desde compositores e letristas, até as gravadoras e os criadores de imagens.

\section{"Geografia e música" nO Debate: Geografia}

TRADICIONAL X NEW CULTURAL GEOGRAPHY

A partir das classificações empreendidas pelos dois principais autores no campo da geografia e música, é possível refletir criticamente acerca de suas perspectivas e da inserção do presente estudo nesta, por assim dizer, corrente da geografia cultural. Os estudos que envolvem geografia e música, como é possível perceber, não estiveram alheios às discussões pós-70 ocorridas no âmbito das ciências humanas e sociais e que transformaram a geografia cultural radicalmente, ou seja, que atingiram a raiz da disciplina: o conceito de cultura. Os debates ocorrem, principalmente, em torno de três eixos principais (Hoefle, 1998):

\section{A abrangência do conceito}

2. A importância atribuída à cultura na explicação do comportamento humano

3. Os processos de mudança cultural

Segundo Hoefle (1998), uma das principais discussões em torno deste conceito reside na 
abrangência dos fenômenos sociais entendidos como cultura. Basicamente, têm-se duas visões principais: de um lado, a abrangente, que entende como cultura todo o conjunto de ações humanas, ou seja, cultura seria "tudo aquilo que o homem faz". De outro lado, há a visão restrita, mais criteriosa, que considera a cultura como a parte cognitiva da vida, a atribuição de significados que o homem faz às diversas esferas da vida, por meio de representações simbólicas, constituindo mapas de significados.

Outra questão relevante sobre a cultura reside no grau de importância atribuída a ela como força comportamental da vida humana (Hoefle, 1998). Existem três acepções básicas em torno desta idéia: 1) Determinante, ou determinismo cultural, no qual cultura é entendida como uma entidade supra-orgânica de valores que paira sobre os indivíduos, determinando seus comportamentos, idéias e formação. Esta corrente geralmente está associada à visão abrangente de cultura e influenciou bastante Carl Sauer e a chamada geografia cultural tradicional $_{i}$ 2) Determinada, vinculada à corrente estruturalista e marxista, na qual a cultura seria apenas uma esfera da vida dependente de outras mais básicas à sociedade, como as forças de produção; 3) A forma holística, onde a cultura é concebida como determinante e determinada ao mesmo tempo, isto é, como uma esfera da vida, mas que interage com as esferas econômica, política e social, atuando com o mesmo grau de importância na formação do modo de vida de diferentes grupos sociais presentes em um determinado contexto, que atua como reflexo, meio e condição para o seu desenvolvimento. Esta é a perspectiva geralmente associada à chamada geografia cultural renovada.
Os processos de mudança cultural representam o terceiro ponto de discussão entre os cientistas que se debruçam sobre o conceito de cultura. Estes processos são abordados sobre três diferentes vieses: 1) o evolucionismo, de caráter nomotético, entende que a mudança cultural em uma sociedade é um processo de evolução temporal linear, no qual não ocorrem saltos, apenas sucessões de etapas. 2) O historicismo, de caráter particularista, afirma que cada cultura possui uma história particular, uma evolução específica, sem seguir padrões universais. 3) As análises sincrônicas, rompendo com as análises de processos temporais, defendem que a cultura só pode ser entendida em um determinado período de tempo. Não é possível estabelecer etapas de evolução, tampouco comparar culturas distintas (Hoefle, 1998).

A partir desta breve recapitulação das principais discussões em torno do conceito de cultura, é possível uma leitura mais criteriosa da trajetória dos estudos sobre geografia e música, assim como as abordagens de Carney e Kong. Como um legítimo representante da geografia cultural de influência saueriana, George O. Carney, em sua análise, não se preocupa em fazer uma distinção rigorosa de trabalhos que abordam a música a partir das formas materiais pelas quais ela pode se apresentar - mídias de gravação (LPs, K7s e CDs), regiões musicais, músicos, eventos e concertos - de trabalhos que pretendem entender como a música está vinculada à produção, comunicação e consumo de significados, ou seja, adota uma visão abrangente desta manifestação cultural. Além disso, a maioria dos trabalhos empíricos, seus e de outros autores, organizados na sua coletânea The Sound of People and Places (2003) dedicam-se ao estudo de 
localização e difusão musical, evitam generalizações, preferindo uma linha idiográfica ou particularista, procurando delinear "áreas culturais" e preocupando-se com a história local, ao invés de tentar traçar uma história mais ampla. Estas características são típicas do pensamento difusionista, que foi levado para os Estados Unidos por Franz Boas e muito influenciou a Universidade da Califórnia em Berkeley a partir de Kroeber, Lowie e Sauer (Hoefle, 1998).

Lily Kong, por ter produzido seus trabalhos sobre geografia e música a partir da segunda metade dos anos 90, sofrendo portanto forte influência pós-modernista, apresenta em seu texto uma preocupação muito maior com a distinção entre trabalhos característicos da geografia cultural tradicional e da geografia cultural renovada. Sua proposta de trabalho, vista anteriormente, dedica-se essencialmente a questões que envolvem conceitos como símbolo, significado, discurso e identidade, preocupando-se com a dimensão cultural em si mesma, embora admita o inter-relacionamento entre as esferas econômica e política, de acordo com a visão holística de cultura. Além disso, entende a música como um "texto", isto é, não como uma unidade fechada, com um sentido único imposto pelo autor, mas sim como um "espaço multidimensional", aberto, fragmentário, inacabado e incoerente, receptivo a múltiplas interpretações concorrentes, reconhecendo a idéia de polivocalidade ou polissemia (Brosseau, 2007).

A intenção desta seção do artigo não foi tentar eleger qual a melhor corrente de pensamento, ou mesmo tentar denegrir a geografia cultural tradicional ou saueriana. Não existe dicotomia, hierarquia, e nem mesmo rivalidade entre as geografias culturais tradicional e renovada. Ambas realizam um grande esforço na busca do entendimento da espacialidade da cultura, mas por meio de questões diferentes e abordagens diferentes. $\mathrm{O}$ que se pretendeu foi apenas sistematizar o conhecimento de forma que a tarefa de encontrar a inserção do sub-campo "Geografia e Música" nas discussões mais amplas da disciplina seja possível e esteja apresentada de forma mais clara.

\section{ConsideraÇõES FINAIS}

Como foi apresentado na segunda seção deste artigo, os trabalhos pioneiros realizados a partir do fim dos anos 60 até a década de 1980 estiveram concentrados principalmente na geografia norte-americana, alcançando a Europa posteriormente. No Brasil, as poucas iniciativas ocorreram a partir dos anos 90 e se desenvolvem ainda muito timidamente. Além disso, autores como George Carney e Lily Kong, entre outros, reconhecem a variedade enorme de vieses possíveis que a música oferece para o geógrafo. Esta é uma visão praticamente consensual. Infelizmente, essa variedade ainda reside muito mais nas "propostas" e "agendas" de pesquisa do que em pesquisas efetivamente realizadas.

Por outro lado, o futuro delineia-se aparentemente promissor. Em um contexto onde se incentiva cada vez mais a interdisciplinaridade, a pesquisa científica objetivando o estudo de manifestações artísticas, que atraem já há algumas décadas profissionais das ciências sociais e humanas, também despertam a curiosidade dos geógrafos de maneira cada vez mais significativa e que, por sua vez, se unem àqueles buscando desenvolver mecanismos conceituais que permitam melhor abordá-las. 
Sobre geografia e música, ainda há muitos caminhos a serem explorados e muitas questões a serem levantadas. Por exemplo, sobre as possibilidades de se abordar a música, não a partir das letras de canções, do mapeamento de áreas musicais, da indústria fonográfica, etc., mas sim do elemento fundamental da qual a música se compõe: o som. Seria possível uma abordagem que busque interpretar a geograficidade da música através da sua estrutura melódica, harmônica ou rítmica, por exemplo? Que conceitos seriam mais adequados para se lidar com uma linguagem sonora? Quais os limites que esta linguagem impõe ao intérprete? Qual metodologia seria mais apropriada? Estas, entre outras, são perguntas ainda carentes, não de respostas prontas, pois diversas respostas, muitas vezes conflitantes, surgem no interior de um debate científico, mas principalmente de atenção por parte dos geógrafos.

\section{NOTAS}

Mestrando do Programa de Pós-Graduação em Geografia da Universidade Federal do Rio de Janeiro.

Email:daniel_ufrj@hotmail.com

REFERÊNCIAS BIBLIOGRÁFICAS ANDERSON, B. Imagined Communities. London: Verso, 1983 BROSSEAU, M. O romance: outro sujeito para a geografia. In: Corrêa, R. L. \& Rosendahl, Z. (org.) Literatura, música e espaço. Rio de Janeiro: EdUERJ, 2007.
CONNELL, J. \& GIBSON C. Sound track: Popular music, identity and place. London: Routledge, 2002

CONNELL, J. \& GIBSON C. World music: deterritorializing place and identity. Progress in Human Geograpby, University Colorado, 28 (3), p. 342-361, Junho 2004

CARNEY G. O (org, The sounds of people and places: A geography of american music from country to classical and blues to bop. Lanham: Rowman and Littlefield, 2003.

CORRÊA, R. L. Geografia, literatura e música popular: Uma bibliografia. Espaço e Cultura, UERJ, Rio de Janeiro, 6, p. 63 67, 1998

CORREAA, R. L. \& ROSENDAHL, Z. (org.) Literatura, música e espaço. Rio de Janeiro: EdUERJ, 2007.

GUIU, C. (org.) Geograpbie et cultures, 59, Université Paris IV - Sorbonne,Paris, 2006

HOEFLE, S.W. Cultura na história do pensamento científico Revista da Pós-Graduação em Geografia. Rio de Janeiro: UFRJ 2 (2), p. 3-30, 1998

KONG, L. Popular Music in Geographical Analysis. Progress in Human Geography, University Colorado, 19 (2), p. 183-98, Junho 1995

LECHAUME A. Chanter le pays: sur le chemins de la chanson quebecoise contemporaine In: Géograpbie et cultures, 21, p. 45-58, Março 1997.

MARCELINO, M. M. Uma leitura do samba rural ao samba urbano na cidade de São Paulo. Dissertação (Mestrado em Geografia). Departamento de Geografia, USP, 2007.

MELLO, J. B. F. O Rio de Janeiro dos compositores da música popular brasileira: 1928-1991. Uma introdução à geografia humanista. Dissertação de Mestrado. Departamento de Geografia, UFRJ, 1991.

MESQUITA, Z. A Geografia Social na Música do Prata. Espaço e Cultura, UERJ, Rio de Janeiro, 3, p. 33-41, 1997.

NASH, P. H. \& CARNEY G. O. The seven themes of music geography. The Canadian Geographer, The Canadian Association of Geographers, Canadá, 40 (1), p. 69-74, Março de 1996.

PESSOA DE BARROS, J. F. Mito, memória e história: a música sacra. Espaço e Cultura, n 9 e 10, p. 35-48, 2000

RIBEIRO, C. R. V. Espaço-vivo: as variáveis de um espaço-vivo investigadas na cidade de Diamantina, do ponto de vista dos músicos. Tese de Doutorado. Departamento de Geografia, PUC-MG, 2006.

SMITH, S. J. Beyond geography's visible worlds: a cultural politics of music. Progress in Human Geograpby, N.21 (4), p. 502-529, abril 1997

VILANOVA NETA, M. A. Recife, a abordagem geográfica das composições musicais de Chico Science. Monografia (Graduação em Geografia). Departamento de Geografia, UFRJ, 2002.

\section{ABSTRACT}

THIS PAPER PRESENTS THE MAIN STUDIES ON THE RELATIONSHIPS BETWEEN CULTURAL GEOGRAPHY AND MUSIC DURING THE LAST FOURTY YARS. ADDITIONALLY, IT PRESENTS ALSO THE CONTRIBUTIONS OF THE TWO MAIN GEOGRAPHERS DEALING WITH GEOGRAPHY AND MUSIC, ARTICULATING THEM TO THE DISCUSSIONS BETWEEN THE TRADITIONAL CULTURAL GEOGRAPHY AND THE NEW CULTURAL GEOGRAPHY. KEYWORDS: CULTURAL GEOGRAPHY, POPULAR MUSIC, GEORGE CARNEY, LILY KONG. 